https://helda.helsinki.fi

\title{
Prenatal sensory development
}

\section{Partanen, Eino}

Cambridge University Press

2017

Partanen , E \& Virtala , P 2017 , Prenatal sensory development . in B Hopkins , E Geangu \& S Linkenauger (eds), Cambridge Encyclopedia of Child Development . 2nd ed. edn , Cambridge University Press, pp. 231-241.

http://hdl.handle.net/10138/309497

acceptedVersion

Downloaded from Helda, University of Helsinki institutional repository.

This is an electronic reprint of the original article.

This reprint may differ from the original in pagination and typographic detail.

Please cite the original version. 
Eino Partanen, eino.partanen@helsinki.fi / eino.partanen@cfin.au.dk

MINDLab / Center of Functionally Integrative Neuroscience (CFIN)

Department of Clinical Medicine

Aarhus University Hospital

Nørrebrogade 44

Building 10G, 5th floor

8000 Aarhus C

Denmark

\&

Cognitive Brain Research Unit (CBRU)

Institute of Behavioural Sciences

PO Box 9 (Siltavuorenpenger 1 B)

FI-00014 University of Helsinki

Finland

http://tuhat.halvi.helsinki.fi/portal/en/person/ejpartan

and

Paula Virtala, paula.virtala@helsinki.fi

Cognitive Brain Research Unit (CBRU)

Institute of Behavioural Sciences

PO Box 9 (Siltavuorenpenger 1 B)

FI-00014 University of Helsinki

Finland

http://tuhat.halvi.helsinki.fi/portal/en/person/pvirtala 
Word count (3680-5000): 6748

\section{Normal and abnormal functional prenatal development}

\section{Introduction}

During the last trimester of pregnancy, fetuses develop rapidly and neural connections between the sensory organs and different brain regions associated with sensory processing are formed. During this period, the fetus begins to learn from its surroundings, preparing it for eventual postnatal life. The early intrauterine functional development and learning is very distinct from later postnatal development and learning, as it occurs in the womb of the mother. The results of intrauterine sensory development and learning are seen in, for example, the abilities of newborns to recognize their parents and their native language, which in turn facilitates attachment and language learning. Because sensory functions develop and the fetus begins to learn during pregnancy, abnormalities during gestation or harmful environmental effects may have detrimental effects on the infant. In addition, developmental disorders, such as dyslexia, affect the early development of the nervous system, which can in some cases be seen in, e.g., abnormal functioning of the auditory system after birth.

The studies on prenatal functional development and learning have been predominantly concerned with audition and thus, this entry focuses mainly on audition and prenatal auditory learning. In this entry, the development of sensory functions during pregnancy and the behavioral states affecting fetal responsiveness are described first, followed by an overview of fetal learning mechanisms. We conclude with a brief overview on how the effects of abnormal prenatal development can be seen after birth, and introduce examples of possibly risky intra- and extrauterine environmental factors.

\section{Prenatal development of cortical sensory functions}

During fetal development, the developing central nervous system undergoes several important temporally overlapping steps during its way toward a mature human brain. First, during the nine months of gestation, the human cortex develops a laminar organization as developing cortical neurons in the subplate migrate to form six distinct cortical layers (see, e.g. Kostović \& Judas, 2010). Second, the developing neurons connect to thalamocortical sensory input approximately on the $24^{\text {th }}$ week of gestation, allowing rapid transfer of sensory information to the cortex (e.g. Kostović \& Judas, 2010). Third, the axons between the neurons begin to myelinate, greatly speeding up the rate at which the action potentials travel along them, although the myelination begins at different ages for different brain structures (see, e.g., Yakovlev \& Lecours, 1967). Taken together, the fetal brain starts to form specific functional structures and regions on the cortex that work in unison (Thomason et al., 2014) and may be dedicated to different tasks (such as speech perception; Perani et al., 2011) and senses. Eventually these 
structures become capable of processing complex and conceptual information, which forms the basis for adult human cognitive functions and learning abilities. While the fetal brain begins to form different functional structures on the cortex, the connections between different brain regions are not well developed. Furthermore, studies suggest that cortico-cortical connections begin to form first between different hemispheres while intrahemispheric connections develop later (Perani et al., 2011; Kostović \& Jovanov-Milošević, 2006). It is important to note that prior to the development of the cortex and its' structures, many sub-cortical structures have already matured much further. For example, the auditory brainstem responses become adult like by school age, whereas the cortex develops well into late adolescence and the subcortical structures may, during early development, replicate or be responsible for many functions that are often ascribed to cortical areas (e.g. Chandrasekaran \& Kraus, 2010). However, more complex cognitive functions (such as language or executive functions) are predominantly based on the cortex, and thus this entry predominantly focuses on the development of the sensory functions on the cortex.

It has been suggested that the sensory functions develop on two partly separate developmental trajectories, dubbed activity-dependent development and stimulus-driven development (see, e.g., Anderson \& Thomason, 2013). Activity-dependent development of cortical regions associated with sensory functions has been suggested to be influenced by low-frequency spontaneous neural activity, seen in the EEG recorded from preterm and full-term infants (e.g. Vanhatalo \& Kaila, 2006). While the exact role of the low-frequency spontaneous neural activity in humans is still not entirely clear, it has been suggested to be associated with the maturation of the thalamocortical connections, specifically the extension of the afferent thalamocortical fibres from the subplate to the maturing primary sensory cortices (visual, auditory and sensorimotor) of the cortical plate (Vanhatalo \& Kaila, 2006). In short, activity-driven development may be seen as priming of our sensory systems for exposure to specific sensory stimuli in the future, and this primed neural network is further built up by stimulus-driven development, or sensory experience, where sensory input shapes the cortical architecture and structure. This is evidenced, for example, by tonotopic organization of the auditory cortex: certain areas of the cortex specialize to process sounds of certain pitch, and animal studies have shown that this process is hampered if the sound input is not normal (i.e., white noise instead of meaningful and varying stimuli). The importance of external stimuli for sensory development was also shown by Muir and Mitchell in 1973 by depriving cats of normal visual stimuli and finding that their visual cortices do not then develop normally. Furthermore, fMRI studies in congenitally deaf humans have shown that the auditory cortices may be recruited for the processing of visual stimuli in absence of auditory input (e.g. Finney et al., 2001), showing how cortical structures primed for exposure to specific types of stimuli develop to process stimuli of different modality due to stimulus-driven developmental processes. Thus, it seems that the development of sensory functions has two partially separate developmental trajectories. First, activity-dependent development may be responsible for the development of species-specific neural architecture and forms a basis for the establishment of more mature networks (e.g. Katz \& Shatz, 1996). Second, the stimulus-driven development shapes the fetal brain to process 
specific sounds it is exposed to. The functional development of specific sensory modalities is further discussed below; see Figure 1 for an overview.

$<$ Figure 1 approximately here $>$

Figure 1. An overview including important events of the functional development of different sensory modalities during the fetal period.

\section{Hearing}

Of all sensory modalities, fetal hearing is possibly the most studied, as it develops early: by $20^{\text {th }}-22^{\text {nd }}$ week of gestation the fetal cochlea is functional, after which it can transmit auditory information from the cochlea to the brainstem and via the thalamic nuclei to the cortex (Lecanuet \& Schaal, 1996). Indeed, earliest fetal reactions to sound have been observed on the $24^{\text {th }}$ week of gestation and most fetuses react to external sounds by exhibiting a startle reaction, which is seen as both fetal movement and as a change in fetal heart rate, at the latest by the third trimester of pregnancy (see Lecanuet \& Schaal, 1996, for a review). Further evidence of early development of fetal hearing can be seen in auditory brainstem responses, which have been recorded already from preterm infants born on the $25^{\text {th }}$ week of gestation (Starr et al,. 1977). Although some parts of the fetal auditory pathway are functional by the third week of gestation, the auditory system and pathways are still developing throughout the pregnancy and long after birth, such as some of the pathways associated with language functions (Brauer et al., 2011). This may be due to both immature status of intrahemispheric axonal connections and their myelination status (Paus, 2010; Thomason et al., 2014).

The fetus is also extensively exposed to auditory stimulation during pregnancy: intrauterine sounds, such as maternal heartbeat or bowel movements are clearly audible, but the fetus is also exposed to external sounds (e.g., maternal voice; see Lecanuet \& Schaal, 1996, for a review). However, fetal hearing differs from infant hearing as the fetal ear canal is filled by amniotic fluid and the sounds are most likely transmitted to the ear via bone conduction. Furthermore, the extrauterine sounds are attenuated when they reach the uterus, and high-pitched sounds especially more so than low-pitched ones (Richards et al., 1992). Thus, the fetuses may be primarily exposed to the low-pitched features of sounds, and it has been suggested that the fetal and the newborn auditory discrimination is predominantly based on the low-frequency components of sounds (Spence \& DeCasper, 1987). Although the sounds the fetus is exposed to are attenuated, one sound source is particularly salient for the fetus, and that is the maternal voice. Although the high-frequency components of the maternal voice are attenuated in a similar fashion as other extrauterine sounds, it is much louder because the maternal voice is also transmitted to the fetus internally via bone conduction. Thus, the maternal voice 
and sounds generated by the mother (such as speech, breathing, heart rate, and those from digestive processes) may have a special role in infant auditory development because they are coupled with the events of the surrounding world (see, e.g. Moon \& Fifer, 2000). Thus, it is presumable that the way the mother reacts to external events may cue the fetus to react to them in an adaptive manner.

Due to the attenuation of external stimuli as they reach the uterus, the fetus may have difficulties in discriminating certain types of sounds; for example, it was found that approximately between $55.1 \%$ and $33.5 \%$ of the native language syllables could be correctly identified by adults when the sounds were attenuated to similar degree as they would be in utero (Griffiths et al., 1994). Furthermore, the fetus is exposed to large amounts of background intrauterine noise (such as maternal digestion) within the womb. The amount of intrauterine background noise appears to vary depending on the location, but it seems that it is predominantly composed of low-frequency sounds and does not seem to be more than $60 \mathrm{~dB}$ SPL (see Lecanuet \& Schaal, 1996, for a review). Thus, when the mother is in a calm environment, the background noise is low enough that the fetus is capable of perceiving and potentially learning from the external auditory input even when high-frequency components of the sounds are attenuated.

Studies assessing the functioning of fetal auditory system during the third trimester have been conducted not only by investigating fetal reactions (such as movement or heart rate changes) to sounds, but also by recording the fetal brain's magnetic responses from the cortex to sounds or changes in sounds by means of MEG (see Anderson \& Thomason, 2013, for a review). The results indicate that the developing fetal brain can discriminate sounds and changes in sounds, but the neural responses associated with change detection may vary greatly between individuals. Furthermore, recent studies using fMRI have managed to localize the neural activity associated with sound processing to the developing auditory cortex (Hykin et al., 1999; Jardri et al., 2012). This indicates that the fetal hearing has sufficiently developed by the third trimester that auditory information can be at least rudimentarily processed on the cortex.

\section{Vision}

Although by the 20-22 weeks of gestation the fetus can open the eyelids, making it possible for the visual stimuli to reach the fetal retina, it is not clear to what extent the fetus can actually process visual stimuli. For example, some studies indicate that the primary visual cortex is somewhat undeveloped both in terms of total volume and synaptic density at birth in comparison to other sensory regions of the brain (Huttenlocher, 1990) and some studies suggest that initially visual functions, such as orienting and attention, are achieved by subcortical structures (see Johnson, 2001, for a review). This suggests that at birth, visual cortex is not yet processing much information, but its' role on visual functions becomes more pronounced during the first months of life (Johnson, 2001), during which the volume of

the visual cortex increases drastically (Huttenlocher, 1990). Furthermore, the research on fetal vision offers partially conflicting evidence for fetal visual functions. First, studies investigating fetal visual 
processing by assessing the brain's magnetic responses to visual stimuli (e.g., flashes of light) using MEG have shown fetal cortical activations to visual stimulation already by $28^{\text {th }}$ week of gestation (Eswaran et al., 2002), which may suggest that the fetal brain can process, or at least react to, visual information. For further details, please see Part II, Chapter 8, Fetal and neonatal magnetoencephalography (MEG).

However, these studies have not been able to pinpoint location of the neural activation related to visual stimuli to the visual cortex, due to the limitations of fetal MEG technique. Interestingly, by studying the fetal visual processing using fMRI, it has been suggested that the fetal neural reactions to visual stimuli occur frontally (see, e.g. Fulford et al., 2003), whereas the primary visual cortex is located in the posterior parts of the brain. It has been suggested that the fetal visual pathway and V1 region of the visual cortex are very immature thus not yet ready to process visual information or that the immature visual cortex may not be sensitive to low level light that can reach the fetus (which may be approx. 5\%, Parraquez et al., 1998), whereas other parts of the visual pathway may react more strongly to visual stimulation (Fulford et al., 2003). However, a recent fetal fMRI study by Schöpf and colleagues (2014) has shown a correlation between the fetal eye movements and activation of visual, motor, and orbitofrontal brain areas. Consequently, Schöpf and others (2014) argue, that the fetus may not able to process visual information, but the spontaneous fetal eye motion is due to endogenous activity-driven development, which links functionally different cortical regions associated with visual processing together, preparing the fetal brain for future stimulus-driven development after birth when the newborn is exposed to visual stimuli.

While the fetal visual cortex may not be sufficiently mature to process visual stimuli, or the visual stimuli that reach the fetus are too faint to be detected and processed properly, exposure to light in the uterus may be paramount for the development of the retina. A recent study in rat pups showed that unless exposed to light during late gestation, the retinal vasculature overgrew, disrupting the normal development of the retina and possibly giving rise to drastic visual dysfunctions (Rao et al., 2013).

\section{Touch, smell and taste}

Fetuses begin to move very early in development and first movements have been observed by $7^{\text {th }}$ week of gestation; such spontaneous movement has been suggested to be indicative of early neural activity (e.g. De Vries et al., 1982). Furthermore, the appearance of different types of movement patterns seems to follow a specific timeline, with startle reactions appearing early in development, followed by isolated movements of the limbs while movement of the head or the mouth appear later (De Vries et al., 1982; De Vries \& Fong, 2006). Furthermore, by studying aborted non-viable fetuses' reactions to touch stimuli immediately after abortion (Hooker, 1952) it has been found that fetuses can, in some cases, respond to touch on the $7^{\text {th }}$ week of gestation. However, for many parts of the body, the nerve endings responsible for registering somatosensory stimulation have not developed until approximately $13^{\text {th }}-14^{\text {th }}$ 
week of gestation (for a review, see Lecanuet \& Schaal, 1996). Even though the somatosensory information can be registered early, it does not reach the developing cortex until approximately $24^{\text {th }}$ week of gestation when the first thalamocortical connections are formed. Nevertheless, the widely accepted view is that fetal movements are predominantly spontaneous, not merely reflexes (see De Vries \& Fong, 2006, for a review). It has been suggested that around the $25^{\text {th }}$ week of gestation fetuses start to actively and spontaneously change position and kick in the utero (Elliot \& Elliot, 1964), possibly due to improved ability to react to somatosensory stimulation and due to the development of the vestibular system to adult-like form, enabling fetuses to more accurately sense their orientation and react to maternal activity. The vestibular system also plays a role in regulating the autonomic system in mammals (for a review, see Jamon, 2014), and has been shown to, for example, play a role in breathing (e.g. Yates, 1996).

Smell (olfaction) and taste (gustation) may develop earlier than other senses. Smells are transmitted via the olfactory bulbs into the olfactory sulci in the brain; the olfactory bulbs are evident approximately between $7^{\text {th }}$ and $8^{\text {th }}$ week of gestation, whereas the olfactory sulci can be seen in the $16^{\text {th }}$ week of gestation (see, e.g., Azoylay et al., 2006). It has been suggested that the olfactory system is sufficiently mature to function by the end of the first trimester of pregnancy and that near-term fetuses' ability to smell is similar to that of neonates (for a review, see Schaal et al., 2004). Studies on fetuses' ability to taste (gustation) are few in number, but the studies on sheep fetuses have suggested that fetuses can taste at latest by the last trimester of pregnancy; however, there may be differences in the developmental trajectories of different types of taste buds (Mistretta \& Bradley, 1978).

It is important to note that our senses of smell and taste are tightly intertwined as some neurons on the olfactory cortex respond to taste stimuli in rats (Maier et al., 2012); in humans, similar effects have been found in fMRI studies (Araujo et al., 2003), or even prior to the cortex in the basal forebrain and insula (Small et al., 1997). In utero, the olfactory and gustatory stimulation both are mediated via the amniotic fluid and its' composition varies based on, for example, the maternal food intake (Schaal et al., 1998); it thus may be that stimuli of these modalities are processed in a multimodal fashion very early in development. Furthermore, the early development of smell and taste may play a role in facilitating attachment between the newborn and the mother, as the newborn can recognize the mother on basis of smell only (for a review, see Moon \& Fifer, 2000). Furthermore, the early development of smell and taste may be beneficial in, for example, preterm care where the familiar odors might be beneficial in alleviating the stress of the preterm infant while separated from the mother (Schaal et al,. 2004). Please also see Part IV, Chapter 12, olfaction and gustation, for a more detailed overview of the development of olfaction and gustation.

\section{Multisensory perception}

In many cases the fetus is not exposed to extrauterine stimuli in a unimodal fashion. This is probably the case especially with auditory stimulation. For example, music spontaneously makes the listeners 
move (Keller \& Rieger, 2009) and thus, music may not be perceived solely as an auditory stimulus, but as a multimodal stimulus where rhythmical movements of the mother are associated with certain changes in the music. Consistent with this, fetal breathing has been shown to change during the last trimester of pregnancy when the mother dances (Hatoum et al., 1997), indicating that the fetus is reacting to maternal exercise or changes in maternal heart rate (Patrick et al., 1982). In similar vein, it has been suggested that maternal speech might be perceived in a multimodal fashion by the fetus as the mother changes her posture or moves while speaking (Moon \& Fifer, 2000). Furthermore, when the mother speaks, the voice may be accompanied by, for example, movement of the diaphragm and abdominal muscles, which may also be perceived by the fetus. These movements may be associated with rhythmical features of speech, and thus could even be used by the fetus to segment auditory stimuli on bases of somatosensory stimulation, promoting native language learning.

Enhancement of learning by multisensory stimulation has been proposed in a theory called intersensory redundancy hypothesis, originally presented by James J Gibson (1904-1979) in 1966. When fetus or infant is not familiar with the stimuli it is exposed to, the simultaneous auditory and somatosensory stimulation may make certain sound features more salient, making it easier for the infant to attend to them and thus facilitating their processing than if the stimuli were presented in one sensory modality only (see, e.g. Bahrick et al., 2004). Consequently, studies in infants have shown that the multisensory stimulation combining especially movement and audition may be beneficial in enhancing learning. For example, Phillips-Silver and Trainor (2005) trained 7-month old babies to recognize certain beats in music by either listening only, listening and seeing an adult move to the beat, or listening and being bounced to the beat themselves. Their results suggest that the learning of the beat was enhanced when the babies were themselves bounced to the beat.

Neurally, cells that respond to more than one sensory modality can be found on the cortex, but also in the superior colliculus. However, it is not known whether different sensory modalities in the human nervous system develop separately or if the young nervous system is inherently multisensory and different senses segregate later in development. Studies in monkeys indicate that the superior colliculus contains a significant number of multisensory neurons immediately after birth, suggesting that even prior to birth monkeys may have the neural architecture to process multimodal stimuli; however, it seems that the multisensory neurons in the newborn monkeys do not give rise to significant response enhancement or response depression - a characteristic typical of adult multisensory neurons (for a review, see Wallace, 2004). It has thus been suggested that sufficient postnatal sensory experience is needed for multisensory integration to mature (Wallace, 2004). However, also opposing results have been found in birds, where the bobwhite quail embryos' learning of maternal call was enhanced by multimodal prenatal simultaneous exposure of the maternal call and patterned light (Jaime et al,. 2010).

Thus, it is not known whether human fetuses’ learning is enhanced by multisensory exposure to stimuli in utero, nor whether multisensory stimulation is essential for the normal development of 
sensory systems. Nevertheless, the newborn nervous system seems to demonstrate considerable crossmodal plasticity. This has been shown in ferrets with neonatal lesions, resulting in neural projections from the retina to divert to the auditory cortex via the auditory thalamus. As a result, the auditory cortex still retains some of the original function, but induces multisensory processing as inputs of auditory and visual information connect to same neurons on the cortex. (e.g., Mao et al., 2011; Mao \& Pallas, 2013).

\section{Fetal behavioral states and the influence of behavioral states to sensory functions}

The behavior and activity of the fetus can generally be classified into four distinct behavioral states: active awake, quiet awake, quiet sleep, and active sleep (Nijhuis et al., 1982). These behavioral states can be understood as distinct and stable states of the fetal sleep-wake cycle, and characterized by distinct pattern of body movements, eye movements, and heart rate (Nijhuis et al., 1982). Fetal behavioral states have been suggested to develop during the last trimester of pregnancy and depending on the interpretation of the data, the fetal behavioral states can be accurately classified by $28^{\text {th }}$ (DiPietro et al., 1996) or $36^{\text {th }}$ week of gestation (Nijhuis et al,. 1982).

Earlier studies on newborn infants suggested that neural processing of auditory stimuli, recorded using EEG, may be influenced by the behavioral state (or the sleep stage) of the newborn (Friederici et al., 2002). However, the effect is not found in all studies (e.g. Martynova et al., 2003) or differences between the states are only minor (Cheour et al., 2002); furthermore, a large majority of studies on neural processing of stimuli assess infants or fetuses in one behavioral state only, or do not differentiate between various behavioral states. It is thus possible, that the behavioral state of the fetus may also influence sensory processing and learning. Interestingly, a recent study indicated that auditory stimulation both increases the amount of time the fetuses spend in active wakefulness and decreases the time spent in quiet sleep; furthermore, the study showed that the latency of fetal auditory evoked cortical responses, measured using fetal MEG, shortened when the fetus was in a more active state (Kiefer-Schmidt et al., 2013). This suggests that fetal sensory functioning is associated with fetal behavioral state, and that more active and attentive behavioral state of the fetus may facilitate processing of stimuli.

\section{Prenatal learning}

Prior to discussing findings on prenatal learning, it is needed to assess what is meant by prenatal learning. It can be claimed that the findings on prenatal learning are indicative of habituation only, with habituation being the simplest example of learning and defined as an attenuation of a behavioral or neural response to a repeating stimulus (James, 2010). However, studies have assessed the effects of long-term prenatal exposure to specific stimuli (such as a particular piece of music) on fetal reactions after birth. If a group of infants exposed to the stimulus in utero shows a preference for the same stimulus after birth whereas a control group unexposed to the stimulus does not, or if the newborn brain responses, recorded using EEG or MEG, differ between the exposed and the unexposed group, it is 
then interpreted that this difference between the groups is indicative of prenatal learning. Thus, it can be argued that the difference between the groups is due to the fetuses forming a (neural) memory trace for the stimulus, which they can recognize after birth. In general, learning effects found in exposure studies are often not considered being due to simple habituation, as the effects of learning are not seen only as diminishment of a response to a stimulus, but as preference of one stimulus over another or as a heightened neural or behavioral response to a familiar stimulus. Furthermore, when assessing prenatal learning on basis of infant behavior after birth, it is important to note that in some cases the processing of complex features may have a biological basis. For example, a recent study suggested that processing of musical intervals in Western music may be based predominantly on the physiological properties of the cochlea (Blinowska et al., 2013).

Prenatal learning has been studied predominantly from the perspective of audition. The reason for this is that fetuses are extensively exposed to extrauterine sounds during development, sounds easily travel long distances, and neural responses to sounds can be recorded in most fetuses (see, e.g. Sheridan et al., 2010). As to the function of prenatal learning, several authors have suggested that prenatal learning enables the infant to orient to the relevant stimuli for attachment and learning (such as the voices of the parents, own native language) and to feel safe or familiarize themselves with the environment they are expected to live in after birth. In short, the role of the fetal learning may be to enable the newborn to distinguish its caregivers and safe environmental events from novel, surprising and possibly threatening (e.g. Moon \& Fifer, 2000; Huotilainen 2010). One well-known example of familiarization to the environment is a Japanese study assessing the reactions of newborns to airplane noise. It was found that the infants whose mothers had been living near an airport and exposed to airplane noise during pregnancy slept more soundly, awakened and cried less when exposed to the noise than the infants for whom the airplane noises were novel (Ando \& Hattori, 1970). Furthermore, newborn infants have been shown to have a preference for native language phonemes over non-native ones, suggesting that fetuses have learned to make this distinction prior to being born (Moon et al., 2013; while still being able to discriminate nonnative phoneme contrasts, see, e.g., Werker \& Tees, 1999).

Fetuses do not only seem to learn from their environment, but they seem to be able to use what they have learned to generate specific actions after birth. A startling example is the study showing that newborns tend to cry using their native language prosody: German infants' cry had a descending melody whereas French infants' cry was ascending (Mampe et al., 2009). This suggests that the role of prenatal learning is not only to enhance receptive capabilities, but also to prepare the fetus to react and respond (by voicing and preferring the native language) in culture-specific manner.

In terms of responding in a culture-specific manner, the newborn preference for culture specific stimuli should not be taken as an inability to discriminate or process unfamiliar stimuli. Instead, prenatal learning can be understood as a part of a continuum where infant perception changes over time from universal to culture-specific. The newborns slowly become neurally committed or enculturated to 
the sound environment they are growing up in (Kuhl, 2004; Hannon \& Trainor 2007), but for a long while infants retain the ability to quickly learn to discriminate non-native sound contrasts if they are exposed to them while for adults the ability to learn is slow and may require extensive training (Hannon \& Trehub, 2005). This suggests that the infant's neural apparatus has not yet been 'locked in' to specifically process stimuli of certain type, such as the speech sounds of the infant's native language.

However, fetal learning is not limited to learning of culture-specific stimuli. Indeed, recent studies have shown fetal learning effects by exposing a group of fetuses to specific stimuli that normally developing fetuses are not exposed to. For example, the study of Partanen et al. (2013a) indicated that newborns prenatally exposed to novel speech sounds, namely pitch increments in the middle of a pseudo word, that are not normally present in the native language of the parents (Finnish), could neurally discriminate such changes whereas fetuses unexposed to these changes could not. A further study indicated that for a melody, newborns and 4-month-old infants prenatally exposed to the melody elicited larger neural responses to the notes in the melody than unexposed fetuses (Partanen et al,. 2013b). Further evidence of long-term effects of fetal learning was found in a French study utilizing discrimination of melodic contours after birth: the fetuses prenatally exposed to a descending piano melody displayed a significant change in heart rate at the age of six weeks although they were not exposed to the melodies after the prenatal stimulation (Granier-Deferre et al., 2011).

Although many studies have clearly shown effect of fetal learning, the factors facilitating such learning are not well known. First, the attenuating effects of the uterus may make certain stimuli undetectable in the womb, and thus the fetus may find some stimulus features more salient than others. Second, it is not known how much fetal learning is enhanced by repetition or whether there is a ceiling effect: in the studies of Partanen et al., (2013a, 2013b), prenatal stimulation lasted for three months whereas in the study of Granier-Deferre et al. (2011) the fetuses were exposed to the melody for three weeks only. Third, it is not known to what extent the fetal learning effects generalize. For example, if the fetuses are exposed to sounds that require accurate pitch perception in order to be discriminated from each other, and if the fetus learns to accurately perceive pitch changes in those sounds, can the fetus then use these abilities to better perceive changes of pitch in general, or only for the specific stimuli the fetus was exposed to in utero? However, at least some level of generalization is illustrated in studies showing that infants are able to process relative sound features: for example infants who have been exposed to a certain melody, recognize that melody even when it is transposed to a different frequency level (Plantinga \& Trainor, 2005).

No need for 'fetal education'

Although fetal learning effects have been shown in various studies and some studies show signs of long-term neural or behavioral effects of fetal learning, nothing yet has suggested that intentional fetal exposure to stimuli could provide any cognitive long-term benefits. Previous studies or training programs aimed to provide cognitive benefits by various means during the pregnancy, such as fetal 
exposure to sounds or certain types of games involving touching the fetus, have not been shown to provide any benefit over normal development, or leaving the fetus to its own 'devices' (for a review, see Moon \& Fifer, 2000). While some studies in animals have shown 'cognitive' benefits (i.e., improved ability to navigate a maze in rats; Aoun et al., 2005) from enriched auditory environment during pregnancy, human fetuses of healthy mothers are normally exposed to very rich environment; indeed, even normally hearing infants whose mothers are deaf (resulting in little or no exposure to maternal voice for the fetus) develop a spoken language (akin to bilinguals, but with certain bimodal features; see Lillo-Martin et al., 2014, for a review).

While it seems unlikely that additional fetal exposure to stimuli could produce cognitive benefits, it has been suggested that parents who are interested in the well-being of their future child could be more eager to try to expose their children to additional prenatal stimulation (Moon \& Fifer, 2000). The interest of the well-being of the child already prior to birth could plausibly be associated with more secure attachment between the child and parents, which has been shown to have cognitive benefits (Moss \& St-Laurent, 2001). This is due to the fact that when an infant or a child is securely attached to its' parents, the child has to spend less cognitive resources to focus on making sure it is safe and it is being taken care of, allowing those resources to be used for exploration, play, and learning instead. Thus, it may be that any anecdotal evidence of cognitive benefits due to additional fetal exposure could be due, in turn, to secure attachment between the parents and the child, and the resources available to the parents that can promote children's well-being and normal development throughout childhood.

While it seems that additional fetal exposure does not enhance cognition, it may have harmful effects. Some stimuli, such as loud noises or bright lights, possibly coupled with maternal stress reactions, might distort the fetal sleep-wake -rhythms or harm the developing nervous system.

\section{Risk factors for prenatal development}

Normal development can be disrupted by several environmental or genetic factors. First, the pregnancy might terminate early, resulting in preterm birth with its own set of complications, and preterm birth seems to have a strong genetic predisposition (for a review, see Crider et al., 2005). Second, the fetus might be exposed to harmful stimuli or substances during development, which may have consequences

for functional development. Third, maternal stress can influence fetal development. Finally, development may by hindered by developmental disorders with a genetic basis that influences early neural development, such as dyslexia (see, e.g., Becker et al,. 2014; Chandrasekar et al., 2013), which are outside the scope of this entry.

In terms of functional development, preterm birth drastically alters the environment that the fetus develops in. In the uterus, the fetus is exposed to a variety of stimuli, but in a different manner than after birth, as discussed in detail previously. After the birth, the preterm infant spends most of its time in an incubator in neonatal intensive care unit, where it is exposed to very different types of 
auditory stimuli, containing, for example, much more high-frequency machine-based noise than in the uterus, especially in the frequencies of the first and second formants, important for vowel identity discrimination (Lahav, 2014).

Preterm birth is associated with a risk of various cognitive deficits, partly due to underlying brain injuries arising from perinatal hypoxia, or abnormal neural development of particularly susceptible neuron types (for a review, see Salmaso et al., 2014). Cortical or subcortical injuries associated with preterm birth might be associated with abnormal development of sensory functions, which are later seen as abnormal cognitive performance that persist well into the childhood (Aylward, 2002). Various treatments have been developed to help with complications associated with preterm birth, which may, however, result in short-term gains in cognitive performance (for a review, see Orton et al., 2009). However, novel interventions to combat the possible deficits associated with preterm birth have been developed, such as kangaroo care where the preterm is held skin-to-skin with an adult. Its many benefits include promoting attachment by being in skin contact with the parent, and some studies have even reported improved cognitive development at the age of 5 and 10 years (Feldman et al., 2014).

The exposure to harmful stimuli during development is not limited to chemical exposure only, although disorders such as fetal alcohol syndrome are quite prevalent. In terms of functional development it seems that, for example, exposure to loud noises can disrupt the normal development of the auditory system. Such an effect was seen in rat pups whose the mothers were exposed to continuous noise during pregnancy (e.g., Chang \& Merzenich, 2003). Due to noise exposure, the rat pups' auditory cortex did not develop normally and organize tonotopically, and the effects of this abnormal development took some time to correct. While human fetuses are not normally exposed to constant noise, the required amount of noise exposure that might disrupt development is currently not known. Thus, it may be that some mothers working in noisy factory or retail environments might be at risk for delayed or compromised fetal auditory system development.

Furthermore, maternal stress, measured both using qualitative descriptions and use of anxiety inventories, during pregnancy has been shown to affect fetal development (for a review, see Van den Bergh et al., 2005). The effects of maternal stress have been suggested to be mediated either by the transfer or hormones, namely cortisol, across the placenta or by impaired blood flow in the uterus due to stress (Van den Bergh et al., 2005). Maternal stress may hinder the development of various brain structures, namely the HPA-axis, the limbic system, and the prefrontal cortex, but it has also been shown that infants of stressed mothers are more difficult to soothe in infancy (Martin et al., 1999). This, in turn, may affect the early development of the child who becomes more restless, having less time for play, exploration, and a steady sleep-wake rhythm. Furthermore, the parents of infants who are difficult to soothe may experience their infant as more difficult, which in turn might influence their attachment with the child. 


\section{Conclusions}

While recent studies have clearly indicated that sensory functions develop in utero and allow for learning to occur, we do not know whether the fetal brain drastically benefits from stimulus-driven development or if endogenous activity-dependent development is sufficient for normal development. Furthermore, our knowledge on the role and limitations of fetal learning is quite limited. For example, are there limits to fetal learning competencies in terms of physical characteristics of the stimuli or how complex can the stimuli be for the fetus to be able to discriminate between them? Furthermore, it is not entirely clear what the function of fetal learning is in terms of postnatal development; does it merely exist to facilitate attachment and adjustment to life after birth, or are there long-term sensory or cognitive consequences of fetal learning (or lack of it)? Therefore, it is reasonable to ask how essential the fetal exposure to external stimuli is in terms of normal development.

In addition to fetal learning, the studies on the development of human sensory function are extremely important if detectable deficiencies in fetal sensory functions would be predictive of future sensory or cognitive outcomes. However, evidence on deficiencies in early sensory functioning and its' association with future outcome is still very sparse but slowly accumulating, and recent studies have suggested a link between the electric responses of the newborn brain with future outcome in infants atrisk for dyslexia (e.g. Leppänen et al., 2010). Considering that the early identification of risks for abnormal development would allow for, for example, preventive interventions to be started very early in development, future studies will eventually need to assess if sensory functioning in the fetus or the neonate is predictive of future outcome. In addition to being of use in assessing possibilities for early interventions, long-term follow-up studies will provide us with more information on the plasticity of the infant brain.

See also: Learning theories; Fetal magnetoencephalography (MEG); fetal ultrasonography; Magnetic Imaging (fMRI); Prenatal neural development; Prenatal development of the musculoskeletal system in the human; The status of the human newborn; Audition; Cognitive development during infancy; Learning and memory; Olfaction and gustation; Attachment (early childhood); Brain and behavioural development; Autism; Dyslexia; Fetal alcohol syndrome; Prematurity and low-birthweight.

\section{Further reading}

Benders, M. J., Palmu, K., Menache, C., Borradori-Tolsa, C., Lazeyras, F., Sizonenko, S., ... \& Hüppi, P. S. (2014). Early Brain Activity Relates to Subsequent Brain Growth in Premature Infants. Cerebral Cortex, bhu097. 
Eswaran, H., Haddad, N. I., Shihabuddin, B. S., Preissl, H., Siegel, E. R., Murphy, P., \& Lowery, C. L. (2007). Non-invasive detection and identification of brain activity patterns in the developing fetus.

Clinical neurophysiology, 118(9), 1940-1946.

Tymofiyeva, O., Hess, C. P., Ziv, E., Tian, N., Bonifacio, S. L., McQuillen, P. S., ... \& Xu, D. (2012). Towards the "baby connectome": mapping the structural connectivity of the newborn brain. PloS one, 7(2), e31029. 